\title{
CHEMERIN IS AN INDEPENDENT MARKER OF THE METABOLIC SYNDROME IN A CAUCASIAN POPULATION - A PILOT STUDY
}

\author{
David Stejskala*, Milan Karpisek $^{\mathrm{b}}$, Zuzana Hanulova ${ }^{\mathrm{c}}$, Marek Svestak ${ }^{\mathrm{a}}$
}

\author{
a Department of Laboratory Medicine \& Internal Department, Sternberk Hospital, Jivavska 20, Sternberk, Czech \\ Republic \\ ${ }^{b}$ Department of Human Pharmacology and Toxicology, Faculty of Pharmacy, University of Veterinary and Pharmaceutical \\ Sciences Brno, Brno, Czech Republic \\ c Gnosis s.r.o., Slovakia \\ e-mail:david.stejskal@nemstbk.cz
}

Received: September 26, 2008; Accepted (with revisions): November 11, 2008

Key words: Chemerin/ELISA /Metabolic syndrome/Adipokines/Adipocytes

Aim: Chemerin is a novel adipokine that has been suggested to play an important role in the pathogenesis of the metabolic syndrome. The aim of our study was to evaluate serum chemerin as a marker of the metabolic syndrome and to assess its predictive accuracy in a Caucasian population.

Methods: The study was designed as a cross-sectional study. Anthropometric measurements and serum analyses were done for Body Mass Index, waist circumference, chemerin, insulin, triacylglycerides, total cholesterol, HDLcholesterol, LDL-cholesterol, uric acid, and glucose in 55 non-obese healthy subjects and 181 subjects at risk for the metabolic syndrome. ROC curves were determined and the Chi-squared test was used to analyse the data.

Results: Compared with healthy controls, subjects with suspected metabolic syndrome had significantly higher chemerin serum levels (medians: 266.0 vs. $192.5 \mu \mathrm{g} / 1$; $\mathrm{P}<0.01$ ). After further chemerin adjustment, the difference between the subgroups persisted. Chemerin serum levels correlated with age $(r=0.23)$, serum glucose $(r=0.23)$, HDL-cholesterol $(r=-0.19)$, triacylglycerides $(r=0.22)$, systolic and diastolic blood pressure $(r=0.40 ; r=0.24)$ and the number of metabolic syndrome risk factors $(\mathrm{r}=0.47)$. At a serum chemerin cut-off level of $240 \mu \mathrm{g} / 1$, the presence of the metabolic syndrome was diagnosed with $75 \%$ sensitivity and $67 \%$ specificity.

Conclusion: In conclusion, serum chemerin levels are associated with the characteristics of the metabolic syndrome and could be an independent marker of this disorder in a Caucasian population.

\section{INTRODUCTION}

Adipose tissue is an active endocrine secretory organ which secretes a number of adipokines such as leptin, tumor necrosis factor alpha (TNF- $\alpha$ ), interleukin 6 (IL-6), resistin, fatty acid binding protein-4 (A-FABP), adiponectin, chemokine ligand 2, PAI-1 (plasminogen activator inhibitor-1), adipsin, renin, angiotensinogen and angiotensin I and II . Adipocytes express receptors for some adipokines (e.g. TNF- $\alpha$, adiponectin, leptin) that are reported to modulate lipid metabolism and adipogenesis directly by autocrine and paracrine mechanisms. Adipokines have important roles in the regulation of systemic lipid and glucose metabolism through systemic actions in the brain, liver and muscle. Adipokine secretion and blood level is mostly affected by the degree of adiposity, leading to the hypothesis that dysregulation of proinflamatory and antiinflamatory adipokines secretion in obesity may serve as a pathogenic link between obesity, insulin resistance and cardiovascular diseases ${ }^{1,2}$.

Chemerin, also known as tazarotene induced gene 2 (TIG2) and retinoic acid receptor responder 2 (RARRES2), is a recently discovered adipokine that has been reported to modulate immune system function through its binding to the chemerin receptor (ChemerinR, chemokine like receptor $1, \mathrm{G}$ protein-coupled receptor $)^{1}$. Expression of chemerin and its receptor increases during the differentiation of preadipocytes into adipocytes. Chemerin induces the phosphorylation of extracellular signal -regulated kinases 1/2 (ERK 1/2) and lipolysis in differentiated adipocytes and 3T3-L1 cells, stimulates intracellular calcium release and inhibits cAMP accumulation $^{1-4}$.

Chemerin is secreted as an $18 \mathrm{kDa}$ inactive pro-protein and undergoes extracellular serine protease cleavage of the C-terminal portion of the protein to generate the $16 \mathrm{kDa}$ active chemerin which is present in plasma and serum. It was originally reported to be present in circulation in plasma and serum, respectively, at 3.0 and $4.4 \mathrm{nM}$ concentrations in humans, and 0.6 and $0.5 \mathrm{nM}$ concentrations in mice. Active chemerin is abundant in ascitic fluid from ovarian cancer patients (1.8-7.0 nM) and synovial fluid of patients with arthritis (22 nM). However, tissues or cells responsible for chemerin secretion into these biological fluids have not been established.

Recent findings strongly suggest that white adipose tissue serves as both a primary source of chemerin secretion as well as a target for autocrine/paracrine chemerin signaling. A critical function of chemerin/Chemerin R is to regulate adipogenesis and metabolic homeostasis in 
adipocytes in mice and humans ${ }^{2}$. Expression of chemerin in white adipose tissue is much higher than in brown adipose tissue in lean subjects ${ }^{2}$. Chemerin and ChemerinR knockdown largely abrogate adipocyte differentiation. Thus, chemerin is essential in early differentiation processes and may contribute to or regulate critical early events in adipogenesis. Recent results also indicate that chemerin and ChemerinR could have an important biological role in the formation of white adipose tissue during normal development and in pathological states such obesity $^{2,5}$. Chemerin downregulation during adipocyte maturation results subsequently in lower expression of perilipin, GLUT4 (insulin-regulated glucose transporter), adiponectin and leptin by mature adipocytes. This novel adipokine probably also modulates metabolic pathways in mature adipocytes. In agreement with this, the absence of chemerin expression results in a reduced basal and stimulated rate of lipolysis. On the other hand, nanomolar concentration of chemerin decreases intracellular cAMP (cyclic-adenosin monophosphate) leading to the assumption that chemerin might oppose the lipolytic action of catecholamines by reducing intracellular cAMP (2). In summary, chemerin has a regulatory role in adipogenesis and adipocyte metabolism, and influencing chemerin and ChemerinR signaling might to lead to novel therapeutic approaches in the treatment of obesity, diabetes mellitus type 2 , and cardiovascular diseases ${ }^{2}$.

The aim of our study was to evaluate serum chemerin as a marker of metabolic syndrome by a new ELISA assay and to assess its predictive accuracy in Caucasian population.

\section{MATERIAL AND METHODS}

\section{Subjects}

The study designed to be cross-sectional was approved by the ethics commission of the Sternberk Hospital, Czech Republic. It was conducted from January 2007 to November 2007 and included a total of 55 healthy individuals all of whom were non-obese, not on any medication and non-smokers), and 181 monitored individuals at high risk of the metabolic syndrome of which 122 were without the metabolic syndrome while 59 had it. Patients at risk for the metabolic syndrome were individuals who were monitored or treated for some indicator of the metabolic syndrome (hypertriacylglyceridemia, prediabetes, dyslipidemia, obesity). According to the National Cholesterol Education Program Adult Treatment Panel III (NCEP ATP III), the metabolic syndrome is diagnosed when three or more of the following criteria are found in a subject: 1) waist circumference equal to or greater than 102 $\mathrm{cm}$ in men, or equal to or greater than $88 \mathrm{~cm}$ in women; 2) elevated triacylglycerides (equal to or greater than 1.7 $\mathrm{mmol} / \mathrm{l}) ; 3$ ) reduced high density cholesterol (HDL-cholesterol) (men - less than $1.0 \mathrm{mmol} / \mathrm{l}$, women - less than $1.3 \mathrm{mmol} / \mathrm{l}$ ); 4) elevated blood pressure (equal to or greater than $130 / 85 \mathrm{~mm} \mathrm{Hg}$ or being treated for blood pressure lowering drugs); 5) Elevated fasting glucose (equal to or greater than $5.6 \mathrm{mmol} / \mathrm{l})\left(\right.$ ref. $\left.^{6}\right)$. Exclusion criteria of the study were as follows: acute or chronic systemic inflammation (medical history, CRP> $10 \mathrm{mg} / \mathrm{l}$, leukocytosis, clinical examination), diabetes mellitus treated with peroral antidiabetic drugs or insulin, liver diseases with transaminases levels above 5 times the normal upper limit, kidney diseases with creatinine levels above $130 \mu \mathrm{mol} / 1$ and/or proteinuria exceeding $0.5 \mathrm{~g} / 1$.

\section{Sampling and laboratory determinations}

Anthropometric, clinical, (height, weight, BMI, waist circumference, systolic and diastolic pressures) and laboratory analyses were performed. Fasting blood samples were drawn under aseptic conditions from vena cubiti after several minutes' rest in half-sitting position. Serum samples were separated by centrifugation at $4{ }^{\circ} \mathrm{C}$ at 3000 $\mathrm{g}$ for $20 \mathrm{~min}$ and subsequently used for analysis or frozen at $-80^{\circ} \mathrm{C}$.

Chemerin serum levels were determined in samples stored at $-80{ }^{\circ} \mathrm{C}$ with a commercially available ELISA assay (BioVendor Research and Diagnostic Products, Inc., Modrice, Czech Republic). The limit of detection of the assay was $0.13 \mu \mathrm{g} / 1$, and the intraassay and interassay coefficient of variation $(\mathrm{CV})$ were always less than $7 \%$. The other measurements were performed on the collection day: adiponectin (BioVendor Research and Diagnostic Products, Inc., Brno, Czech Republic), cholesterol, triacylglycerides, high density-cholesterol (HDL), low density-cholesterol (LDL) (Siemens, Advia 1650, Tarrytown, USA), glucose, uric acid, apolipoprotein A-I, apolipoprotein B, alanin aminotranspherase (ALT), aspartat aminotranspherase (AST), insulin (Siemens, Immulite 2000, Tarrytown, USA). Insulin sensitivity check index (Quicki) and Body Mass Index (BMI) were calculated, and waist circumference (WC) was measured.

\section{Statistical analysis}

The data were analyzed using the statistics software package (MedCalc, Mariakerke, Belgium). Values of $p<0.05$ were considered statistically significant. Spearman's correlation coefficients were used to establish the association between chemerin level and other parameters. Comparisons of chemerin serum values between risk subjects with and without the metabolic syndrome were made by either Student $t$ test or Mann-Whitney test according to the distribution of the data. Frequency tables for the chi-squared test for chemerin values and the metabolic syndrome relationship and multiple regression for data adjustment to chemerin were done. Chemerin levels were also compared between risk groups with and without metabolic syndrome by covariant analysis using HDL-cholesterol, triacylglycerides, glucose, sex, age, BMI, WC and Quicki as covariates and adjusted using the linear regression model. The stepwise regression logistic model was also used. All data were expressed as medians or means \pm standard deviations. 
Table 1. Serum chemerin values in healthy subjects $(n=55)$ according sex.

\begin{tabular}{|c|c|c|c|c|}
\hline Parameter & $\begin{array}{c}\text { Chemerin- mean } \\
(\mu \mathrm{g} / 1)\end{array}$ & $\begin{array}{c}\text { Chemerin- median } \\
(\mu \mathrm{g} / \mathrm{l})\end{array}$ & SD & P \\
\hline Women + Men & 186.0 & 192.5 & 25.7 & - \\
\hline Women & 196.7 & 209.0 & 28.3 & - \\
\hline Men & 171.2 & 167.0 & 11.9 & 0.09 \\
\hline
\end{tabular}

P - significance SD - standard deviation

Table 2. Biochemical parameters in subjects with $(n=59)$ and without metabolic syndrome $(n=122)$.

Data presented as median (mean $+\mathrm{SD})$.

\begin{tabular}{|c|c|c|c|}
\hline Parameter & $\begin{array}{c}\text { Metabolic syndrome } \\
\text { present }\end{array}$ & $\begin{array}{c}\text { Metabolic syndrome } \\
\text { not present }\end{array}$ & Value of probability $\mathrm{P}$ \\
\hline Adiponectin (mg/1) & $10.80(11.80+7.20)$ & $10.0(12.80+7.50)$ & 0.71 \\
\hline Age (years) & $62.0(62.40+11.90)$ & $64.0(63.50+12.20)$ & 0.57 \\
\hline $\operatorname{ALT}(\mu \mathrm{kat} / 1)$ & $0.48(0.55+0.30)$ & $0.48(0.61+0.72)$ & 0.85 \\
\hline $\operatorname{AST}(\mu \mathrm{kat} / 1)$ & $0.50(0.55+0.22)$ & $0.49(0.62+0.7)$ & 0.96 \\
\hline BMI $\left(\mathrm{kg} / \mathrm{m}^{2}\right)$ & $31.20(30.60+4.60)$ & $27.10(28.0+4.42)$ & $<0.01$ \\
\hline CK $(\mu \mathrm{kat} / 1)$ & $1.60(2.35+2.37)$ & $1.93(2.28+1.40)$ & 0.09 \\
\hline Glucose (mmol/1) & $8.40(9.05+23.89)$ & $5.55(6.26+2.50)$ & $<0.01$ \\
\hline HDL (mmol/1) & $1.16(1.20+0.30)$ & $1.49(1.53+0.37)$ & $<0.01$ \\
\hline HOMA (-) & $3.97(4.27+2.64)$ & $4.1(4.39+2.78)$ & 0.94 \\
\hline Chemerin $(\mu \mathrm{g} / 1)$ & $266.0(285.0+80.70)$ & $230(228.90+41.10)$ & $<0.01$ \\
\hline Cholesterol (mmol/1) & $4.90(8.20+4.22)$ & $5.2(8.60+4.9)$ & 0.13 \\
\hline Insulin $(\mathrm{kIU} / 1)$ & $10.60(10.93+6.97)$ & $10.5(12.47+8.2)$ & 0.6 \\
\hline Uric acid $(\mu \mathrm{mol} / 1)$ & $354.0(358.0+101,7)$ & $319.5(321,3+73,5)$ & $<0.01$ \\
\hline $\mathrm{LDL}(\mathrm{mmol} / 1)$ & $2.71(2.83+0.74)$ & $2.86(2.96+0.77)$ & 0.27 \\
\hline Quicki (-) & $0.51(0.56+0.18)$ & $0.53(0.63+0.22)$ & 0.2 \\
\hline Blood pressure diastolic (torr) & $80.0(83.1+4.73)$ & $80.0(80.74+3.7)$ & $<0.01$ \\
\hline Blood pressure systolic (torr) & $135.0(136.0+6.94)$ & $130.0(131.0+3.90)$ & $<0.01$ \\
\hline TAG (mmol/l) & $2.06(2.24+0.98)$ & $1.38(1.61+0.84)$ & $<0.01$ \\
\hline $\mathrm{WC}(\mathrm{cm})$ & $101.0(98.86+10.8)$ & $90.0(92.80+15.10)$ & $<0.01$ \\
\hline
\end{tabular}

WC - waist circumference, TAG - triacylglycerides P - significance SD - standard deviation

Table 3. Adjusted chemerin values in risk subjects with and without metabolic syndrome.

Data presented as median (by data distribution).

\begin{tabular}{|l|c|c|c|}
\hline \multicolumn{1}{|c|}{ Parameter adjusted } & $\begin{array}{c}\text { Metabolic syndrome } \\
\text { present }\end{array}$ & $\begin{array}{c}\text { Metabolic syndrome } \\
\text { not present }\end{array}$ & P \\
\hline Chemerin adjusted by BMI & 9.5 & 8.4 & 0.01 \\
\hline Chemerin adjusted by TAG & 162.7 & 146.9 & $<0.01$ \\
\hline Chemerin adjusted by HDL & 223.3 & 138.1 & $<0.01$ \\
\hline Chemerin adjusted by glucose & 34.1 & 39.6 & $<0.01$ \\
\hline Chemerin adjusted by age & 4.8 & 3.7 & $<$ \\
\hline
\end{tabular}

$\mathrm{P}$ - significance 


\section{RESULTS}

In the group of healthy volunteers $(\mathrm{n}=55,26$ men, 24 women), we found no statistically significant difference in chemerin levels between men and women (Tab. 1). In the group of high risk probands, individuals with the metabolic syndrome had significantly different chemerin serum levels compared to individuals without the metabolic syndrome or healthy subjects (abnormal distribution, medians 266, 230, $192.5 \mu \mathrm{g} / 1$ respectively, $\mathrm{P}<0.01$ (Tab. 1, Tab. 2). Neither subgroup of high risk patients (with or without the metabolic syndrome) differed in sex or age but among these subgroups we found correlations between chemerin and age $(\mathrm{r}=0.23, \mathrm{P}<0.01)$, glucose $(r=0.23, P<0.01)$, HDL-cholesterol $(r=-0.19$, $\mathrm{P}<0.01)$, triacylglycerides $(\mathrm{r}=0.22 ; \mathrm{P}<0.01)$, systolic and diastolic blood pressure $(\mathrm{r}=0.40, \mathrm{P}<0.01 ; \mathrm{r}=0.24$, $\mathrm{P}<0.01)$ and the number of risk components of the metabolic syndrome $(r=0.47, \mathrm{P}<0.01)$. No significant correlation was found between serum chemerin and BMI, WC, Quicki (not shown).

The Chi-squared analysis confirmed the relationship between serum chemerin and the diagnosis of the metabolic syndrome (Chi-squared 38.8; df 1; P < 0.01).

The diagnostic efficacy of serum chemerin levels was a sensitivity of $75 \%$ and specificity of $67 \%$ (AUC $0.75 ; 95 \%$ CI $0.68-0.81 ; \mathrm{LR}+2.22)$ at a chemerin cutoff concentration of $240.0 \mu \mathrm{g} / 1$ (Fig 1). The observed diagnostic efficacy of chemerin for the metabolic syndrome $(\mathrm{P}<0.01)$ was better than the efficacy of adiponectin.

After adjustment for age and gender, chemerin levels were significantly associated with metabolic syndrome related phenotypes (fasting glucose, fasting triacylglycerides, fasting HDL-cholesterol) but not with insulin sensitivity (Quicki) or adiposity (BMI, WC). After further adjustment for BMI, serum chemerin levels were still independently associated with metabolic syndrome related phenotypes, including blood pressure, serum glucose, HDL-cholesterol and triacylglycerides. The difference

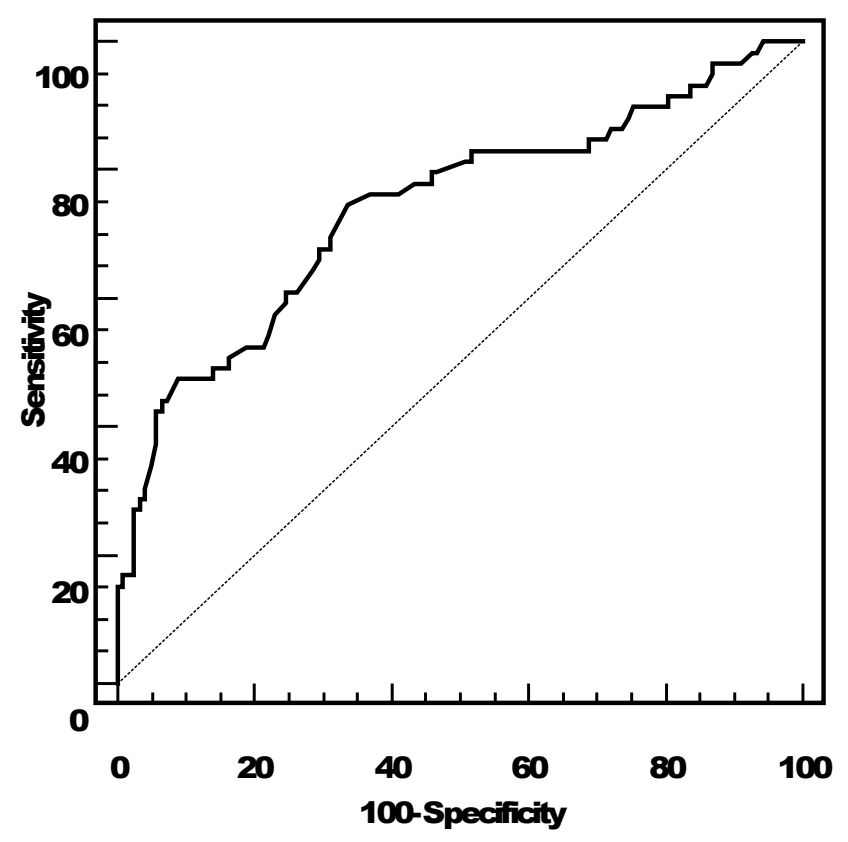

Fig. 1. ROC analysis of chemerin and metabolic syndrome presence.

between both subgroups risk individuals (with or without metabolic syndrome) remained also after chemerin level adjustment for glucose, sex, HDL-cholesterol, age $(\mathrm{P}<0.01)$ but not for triacylglycerides. These results clearly demonstrate that circulating chemerin levels are associated with key characteristics of the metabolic syndrome (Tab. 3). In the stepwise logistic regression model, only chemerin, HDL-cholesterol and glucose were included for metabolic syndrome estimation. Percent of cases correctly classified was 83.3 with an odds ratio 0.03 for HDL-cholesterol, 1.01 for glucose and 1.4 for chemerin.

When patients with the metabolic syndrome were further stratified according to the number of the components of the metabolic syndrome, median (mean+SD) serum concentrations of chemerin for those with 0,1 ,

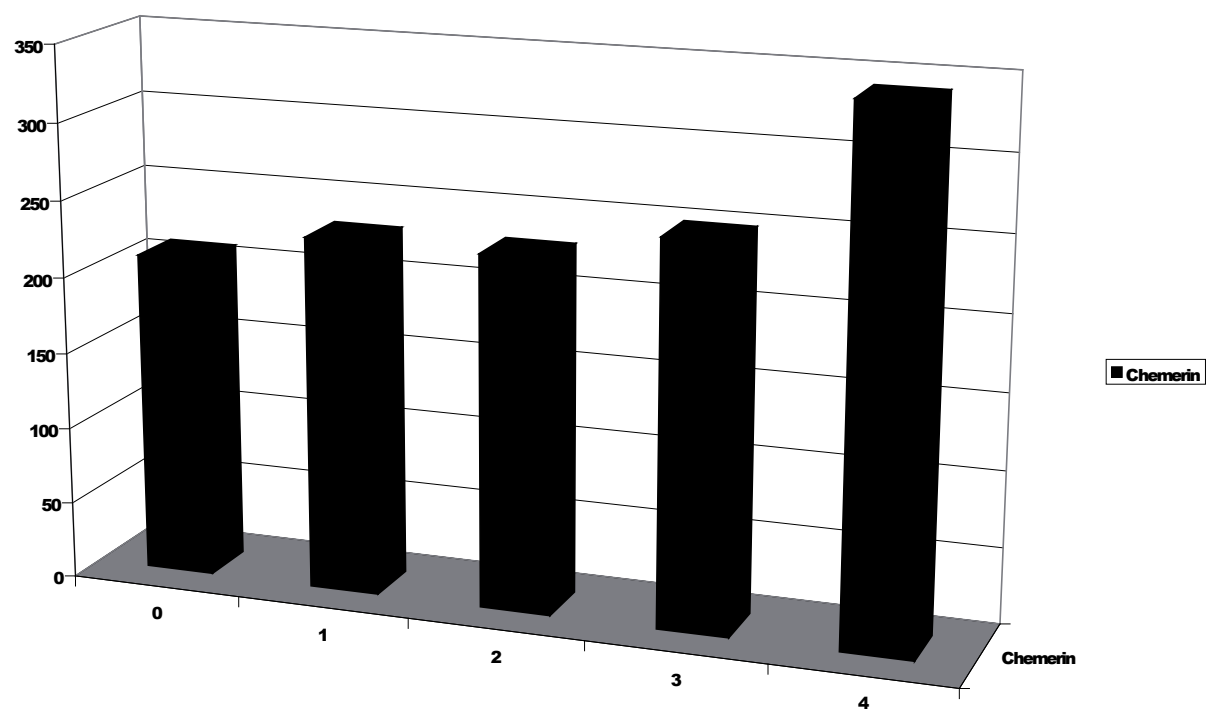

Fig. 2. Serum median chemerin levels $(\mu \mathrm{g} / \mathrm{l})$ stratified according to the number of the components of the metabolic syndrome. 
2, 3 and $>4$ components were $210.0(193.1+40.1) \mu \mathrm{g} / 1$, $231.0(224.9+45.5) \mu \mathrm{g} / 1,230.0(235.7+35.9) \mu \mathrm{g} / 1,249.5$ $(251.6+47.2) \mu \mathrm{g} / 1,341.5(388.5+122.2) \mu \mathrm{g} / 1$ (Fig 2). We found a significant correlation between serum chemerin and the number of metabolic syndrome criteria and this relationship was confirmed by the chi squared test (Chisquare 66.5; $\mathrm{P}<0.01$ ).

\section{DISCUSSION}

The metabolic syndrome is a cluster of metabolic and cardiovascular disturbances, such as central obesity, hypertension, dyslipidemia and hyperglycemia. The source of chemerin protein in circulation is unclear at present. The highest chemerin expression is found in liver, adipose tissue and kidney. As chemerin mRNA expression is found to be increased in adipose tissue but not in the liver of obese compared to lean individuals, it is tempting to speculate that elevated chemerin levels found in plasma of obese humans may originate in adipose tissue ${ }^{5}$. As chemerin is a proinflammatory cytokine activating immune cells, it might play a role in the inflammation of adipose tissue that occurs in obesity. Chemerin protein exists as a full length protein and a short form that is produced by removing 5 amino acids at the $\mathrm{C}$-terminal end of chemerin protein by serine proteases, such as neutrophil elastase, cathepsin $\mathrm{G}$ and plasmin. The full length isoform of chemerin has significantly lower biological activity than the proteolytically processed short form. It is unclear which form of chemerin is found in adipose tissue; however several known chemerin-activating proteases such is $\mathrm{C} 1 \mathrm{~s}$, cathepsin $\mathrm{G}$ are present in adipose tissue. It has been hypothesized that chemerin may be proteolytically cleaved to the bioactive form in the adipose tissue of obese animals, whereas in lean animals, chemerin remains as the inactive full-length form ${ }^{5}$. Serum chemerin values were decreased in $\mathrm{db} / \mathrm{db}$ mice $^{6}$. In addition, chemerin potentiated insulin-stimulated glucose uptake in 3T3-L1 adipocytes concomitant with enhancing insulin signaling. ChemerinR is additionally expressed in macrophages suggesting that chemerin plays a potential role in regulating inflammation by recruiting antigen-presenting cells.

The present study has shown that serum chemerin levels are associated with parameters of the metabolic syndrome and with hypertension regardless of the presence of metabolic syndrome components suggesting that chemerin may also be a novel regulator of blood pressure because of good correlations with both systolic and diastolic pressure. This hypothesis is supported by the fact that chemerin is highly expressed in the kidney, a key site of blood pressure regulation. Chemerin is structurally related to other circulating factors, including cathelicidins, cystatins, and kininogens; the proteolytic product of kininogen is the vasoactive peptide bradykinin. Similar results were recently presented in a large-scale epidemiological study from Mauritius ${ }^{5}$; however, unlike that study, we found no relation between chemerin and adiposity. On the other hand, we detected a previously undocumented and significant relation between serum glucose and chemerin. These differences might be due to the different populations studied 5 .

In conclusion, our study indicates that circulating serum chemerin levels are associated with several key parameters of the metabolic syndrome in a Caucasian population. These findings suggest that chemerin may play a role in the metabolic syndrome and might be an independent promising adipokine marker of metabolic syndrome. Further research is necessary to confirm these findings and to evaluate serum chemerin levels as a predictor of accelerated atherosclerosis or impaired glucose tolerance.

\section{REFERENCES}

1. Roh S, Song SH, Choi KC, Katoh K, Wittamer V, Parmentier M, et al. Chemerin - A new adipokine that modulates adipogenesis via its own receptor. Biochem and Biophys Research Comm 2007; 362:1013-18

2. Goralski KB, McCarthy TC, Hanniman EA, Zabel BA, Butcher EC, Parlee SD, et al. Chemerin, a novel adipokine that regulates adipogenesis and adipocyte metabolism. The $\mathbf{J}$ of Biol Chemistry 2007; 282:28175-88.

3. Wittamer V, Franssen JD, Vulcano M, Mirjolet JF, Poul EL, Migeotte I, et al. Specific recruitment of antigen-presenting cells by chemerin, a novel processed ligand from human inflammatory fluids. J Exp Med 2003; 198:977-85.

4. MacDougald OA, Burant CF. The rapidly expanding family of adipokines. Cell Metabolism 2007; 6:159-61.

5. Bozaoglu K, Bolton K, McMillan J, Zimmet P, Jowett J, Collier G, et al. Chemerin is a novel adipokine associated with obesity and metabolic syndrome. Endocrinology 2007; 148:4687-94.

6. Executive Summary of the Third Report of The National Cholesterol Education Program (NCEP) Expert Panel on Detection, Evaluation And Treatment of High Blood Cholesterol In Adults (Adult Treatment Panel III). JAMA 2001; 285:2486-97. 
